\title{
Sexual Function in Patients with PCOS and/or Obesity before and after Metformin Treatment
}

\author{
Antoaneta Gateva, Zdravko Kamenov \\ University Hospital Alexandrovska, Medical University of Sofia, Sofia, Bulgaria \\ Email: tony_gateva@yahoo.com
}

Received January 10, 2012; revised February 12, 2012; accepted March 1, 2012

\begin{abstract}
The clinical signs of hyperandrogenemia, commonly seen in polycystic ovarian syndrome (PCOS) patients combined with obesity and infertility can cause emotional distress. There are however few data about the psychosocial and sexual function of patients with PCOS. Objectives: The aim of the study was to investigate the sexual function in patients with obesity and/or PCOS using Female sexual function inventory (FSFI) before and after metformin treatment. Design and Methods: In the present study were included 79 patients divided into three groups - group 1 Obese $(\mathrm{n}=22)$; group 2 Lean PCOS $(n=41)$ and group 3 Obese PCOS $(n=16)$. All of the subjects completed FSFI questionnaire. In patients who had insulin resistance (OGTT + IRI) metformin treatment was started in dose 1700 - $3000 \mathrm{mg} /$ day. Results: Obese women without PCOS showed significantly higher scores on total FSFI and all domains except from desire compared to lean PCOS subjects. Although the differences do not reach statistical significance, lean PCOS patients have the lowest scores on all domains. FSFI score correlates negatively only with androstendione levels. Women with and without hyperandrogenemia do not show differences in FSFI score. It is interesting to note that LH but not FSH shows moderate positive correlation to all domains of FSFI. FSFI scores do not show correlation to the indices of carbohydrate metabolism (blood glucose and IRI during OGTT), lipid profile, and arterial pressure. After metformin treatment there was an increase in all FSFI domain scores although statistical significance was noted only for the total FSFI score and the domains lubrication and pain, probably because of the small number of patients. Conclusions: Lean PCOS patients have lower scores on FSFI than obese patients with or without PCOS. The hyperandrogenemia is not a determinant for sexual dysfunction in PCOS women. Metformin treatment has a favorable effect on sexual function.
\end{abstract}

Keywords: PCOS; Sexual Function; Metformin

\section{Introduction}

Polycystic ovarian syndrome (PCOS) is a common endocrine disorder that is characterized by menstrual irregularities, clinical and/or biochemical hyperandrogenism and ultrasound data of polycystic ovaries. The clinical signs of hyperandrogenemia (hirsutism, acne, seborrhea, alopecia) combined with obesity and infertility can undoubtedly cause emotional distress. There are however very few data about the psychosocial and sexual function of patients with PCOS [1]. Anxiety, depression, food disorders and lower self esteem are described. Some authors state that girls with PCOS are 2.8 times less likely to have sexual intercourse than healthy controls [2].

The precise role of androgens in sexual arousal is however controversial and not extensively studied [3]. In fact PCOS women have the same frequency of sexual activity as control subjects [4]. One recent study does not show significant differences in blood flow around the clitoris between patients with PCOS and healthy women [5]. Moderate hirsutism and hyperandrogenism in PCOS pa- tients do not affect sexual self esteem and satisfaction.

Treatment of patients with androgen excess with antiandrogens shows controversial results in a small group of patients with hirsutism ranging from decreased to increased sexual desire [6] while metformin use improves psychosocial, emotional and psychosexual state of the PCOS patients [7] probably due to reduction of clinical symptoms.

Obese PCOS patients probably have increased rate of sexual dysfunction. These data are not confirmed in lean PCOS patients. Additional studies are necessary to determine if normalizing the androgen excess and insulin resistance would improve the sexual function in PCOS patients [8].

\section{Materials and Methods}

In the present study were included patients with PCOS and/or obesity that were recruited from the hospitalized in the Clinic of Endocrinology in Alexandrovska University Hospital in Sofia. 
The following inclusion and exclusion criteria were used:

\section{Inclusion criteria}

$\checkmark$ premenopausal women aged 18 to 45 ;

$\checkmark$ PCOS, diagnosed by ESHRE-ASRM criteria and;

$\checkmark$ BMI $>30 \mathrm{~kg} / \mathrm{m}^{2}$.

Exclusion criteria

$\checkmark$ Pregnancy;

$\checkmark$ Serious illnesses as cardiac, renal or liver insufficiency;

$\checkmark$ Other endocrine pathology like type 2 diabetes mellitus, adrenal tumors, hypothyroidism, prolactinomas, hypogonadism, Cushing's disease, congenital adrenal hyperplasia;

$\checkmark$ Insulin sensitizing medication (metformin or glitazones) or combined oral contraceptive (COC, containing etinylestradiol and progestin) use less than 4 months prior to the study.

The following information for each patient was obtained:

1) General information - name, age;

2) Anthropometric data - height, weight, body mass undex (BMI), waist, hip, waist-to-hip ratio (WHR), waistto-stature ratio (WSR). All measurements were performed when the patients were in a standing position with feet together, relaxed abdomen and arms at their sides. Waist circumference of subjects was measured in standing position by placing a soft tape measure midway between the lowest rib and the iliac crest and hip circumference hip circumference at the level of the great trochanters;

3) Obesity was accepted at BMI $\geq 30 \mathrm{~kg} / \mathrm{m}^{2}$;

4) Polycystic ovary syndrome was diagnosed according to the ESHRE-ASRM criteria - two out of the following: 1) oligo/amenorrhea; 2) clinical or biochemical hyperandrogenism and 3) polycystic ovaries at ultrasound examination when all other endocrine causes are excluded;

5) Results form the oral glucose tolerance test (OGTT) -blood glucose and imunoreactive insulin (IRI) on 0, 60 and $120 \mathrm{~min}$. Homeostatic model assessment (HOMA-IR) was calculated as baseline blood glucose ( $\mathrm{mmol} / \mathrm{L}) \mathrm{mul}-$ tiplied by baseline IRI (mU/1) and the divided by 22.5 ;

6) Hormonal status (testosterone, androstendione, dehydroepiandrosteron sulphate (DHEAS), 17-OH-progesterone, estradiol, LH, FSH, TSH, prolactin). If necessary, all other routine tests were performed to exclude other endocrine pathology as mentioned in the exclusion criteria.

All laboratory tests were performed in the Central Clinical Laboratory of the Alexandrovska University Hospital in Sofia, which is the reference laboratory for the country.

Patients completed Female Sexual Function Index (FSFI) questionnaire [9] that consists of 19 questions, divided in 6 domains-Desire (q. 1, 2), Arousal (q. 3-6), Lubrication (7-10), Orgasm (q. 11-13), Satisfaction (q.
14-16) and Pain (q. 17-19). Score is then formed for each domain separately and a Total FSFI score is then calculated. Full Scale Score Range is from 2.0 to 36.0. Sexual dysfunction was diagnosed at FSFI score $<26$ points.

\subsection{Therapeutic Intervention}

In the patients that had indices of insulin resistance based on OGTT (baseline IRI $>20 \mathrm{UI} / \mathrm{l}$; IRI on $120 \mathrm{~min}>100$ mU/1, HOMA index > 2.0) metformin treatment was initiated and the dose was escalated to $1700-3000 \mathrm{mg} /$ daily. The therapy started with $500 \mathrm{mg}$ in the evening and was increased with $500 \mathrm{mg}$ every 5 - 7 days until final dose was reached. Patients that came back for evaluation completed the same questionnaire after 4 - 12 months of treatment and had all baseline tests repeated.

\subsection{Statistical Methods}

The data were processed using the statistical package SPSS 16.0. The level of significance for rejecting the null hypothesis was $\mathrm{p}<0.05$. The following statistical methods were applied: descriptive analysis, variation analysis, Kolmogorov-Smirnov's one sample non-parametric test, Student's t-test for two independent samples, KruskalWallis' non-parametric test for several independent samples, Mann-Whitney's non-parametric test for two independent samples, one-way analysis of variance betweengroups ANOVA with post-hoc tests, correlation analysis, $\mathrm{ROC}$ analysis. Data are presented as mean $\pm \mathrm{SD}$.

\section{Results}

In the present study were included 79 patients divided into three groups - group 1 Obese $(\mathrm{n}=22)$; group 2 Lean PCOS $(n=41)$ and group 3 obese PCOS $(n=16)$. Comparison was made between the data form these three groups.

Table 1 shows the anthropometric characteristics of the groups. Obese and obese PCOS patients differ signifycantly only in age and have similar anthropometric indices, while lean PCOS patients have lower body weight, BMI, WHR and WSR than the other two groups.

Obese women without PCOS have significantly higher scores on total FSFI and all domains except from desire compared to lean PCOS subjects (Table 2). Although the differences do not reach statistical significance, lean PCOS patients have the lowest scores on all domains except from desire where the lowest score is for obese PCOS women. The rate of sexual dysfunction (total FSFI score $<26$ ) is two times higher in obese PCOS patients and three times higher in lean PCOS patients than in obese patients without PCOS. There is a statistical significant difference $(p<0.001)$ in the rates of sexual dysfunction between obese and lean PCOS patients (Figure 1). 
Table 1. Anthropometric characteristics of the groups.

\begin{tabular}{cccc}
\hline & \multicolumn{3}{c}{ Patients } \\
\cline { 2 - 4 } & $\begin{array}{c}\text { Group 1 } \\
\text { Obese } \\
(\mathrm{n}=22)\end{array}$ & $\begin{array}{c}\text { Group 2 } \\
\text { Lean PCOS } \\
(\mathrm{n}=41)\end{array}$ & $\begin{array}{c}\text { Group 3 } \\
\text { Obese PCOS } \\
(\mathrm{n}=16)\end{array}$ \\
\hline Age (years) & $32.5 \pm 8.5^{\wedge \wedge \wedge}$ & $24.2 \pm 4.8$ & $24.9 \pm 4.4 \# \#$ \\
Height $(\mathrm{cm})$ & $162.2 \pm 6.3$ & $164.8 \pm 6.9$ & $164.1 \pm 6.4$ \\
Weight $(\mathrm{kg})$ & $105.4 \pm 22.8^{\wedge \wedge \wedge}$ & $61.9 \pm 10.5^{* * *}$ & $95.8 \pm 13.4$ \\
BMI (kg/m $\left.{ }^{2}\right)$ & $40.2 \pm 9.4^{\wedge \wedge \wedge}$ & $22.7 \pm 3.4^{* * *}$ & $35.6 \pm 4.9$ \\
Waist $(\mathrm{cm})$ & $111.1 \pm 13.0^{\wedge \wedge \wedge}$ & $77.9 \pm 9.4^{* * *}$ & $104.3 \pm 12.5$ \\
Hip (cm) & $124.6 \pm 11.7^{\wedge \wedge \wedge}$ & $96.7 \pm 8.1^{* * *}$ & $118.3 \pm 7.3$ \\
WHR & $0.89 \pm 0.08^{\wedge \wedge \wedge}$ & $0.81 \pm 0.06^{* *}$ & $0.88 \pm 0.09$ \\
WSR & $0.69 \pm 0.09^{\wedge \wedge \wedge}$ & $0.47 \pm 0.06^{* * *}$ & $0.64 \pm 0.08$ \\
\hline
\end{tabular}

$* \mathrm{p}<0.05 ; * * \mathrm{p}<0.01 ; * * * \mathrm{p}<0.001$ between group 2 and group 3 ; ^ $\mathrm{p}<$ $0.05 ; \wedge \wedge \mathrm{p}<0.01 ; \wedge \wedge$ p $<0.001$ between group 1 and group 2 ; \#\# $\mathrm{p}<0.01$ between group 1 and group 3 .

Table 2. FSFI total score and separate domains scores in the three groups.

\begin{tabular}{cccc}
\hline & \multicolumn{3}{c}{ Patients } \\
\cline { 2 - 4 } & $\begin{array}{c}\text { Group 1 } \\
\text { Obese } \\
(\mathrm{n}=22)\end{array}$ & $\begin{array}{c}\text { Group 2 } \\
\text { Lean PCOS } \\
(\mathrm{n}=41)\end{array}$ & $\begin{array}{c}\text { Group 3 } \\
\text { Obese PCOS } \\
(\mathrm{n}=16)\end{array}$ \\
\hline FSFI & $24.3 \pm 11.7^{\wedge}$ & $15.6 \pm 11.7$ & $17.9 \pm 13.9$ \\
Desire & $3.9 \pm 1.2$ & $3.5 \pm 1.3$ & $3.0 \pm 1.4 \#$ \\
Arousal & $4.1 \pm 2.1^{\wedge}$ & $2.5 \pm 2.4$ & $2.6 \pm 2.4$ \\
Lubrication & $4.4 \pm 2.5^{\wedge \wedge}$ & $2.7 \pm 2.6$ & $3.1 \pm 2.8$ \\
Orgasm & $4.1 \pm 2.3^{\wedge}$ & $2.3 \pm 2.4$ & $2.7 \pm 2.5$ \\
Satisfaction & $4.1 \pm 2.3^{\wedge}$ & $2.6 \pm 2.2$ & $3.4 \pm 2.3$ \\
Pain & $3.7 \pm 2.4^{\wedge}$ & $2.0 \pm 2.5$ & $3.2 \pm 2.9$ \\
\hline
\end{tabular}

$\wedge \mathrm{p}<0.05 ; \wedge \wedge \mathrm{p}<0.01$ between group 1 and group 2 ; $\# \mathrm{p}<0.05$ between group 1 and group 3 .

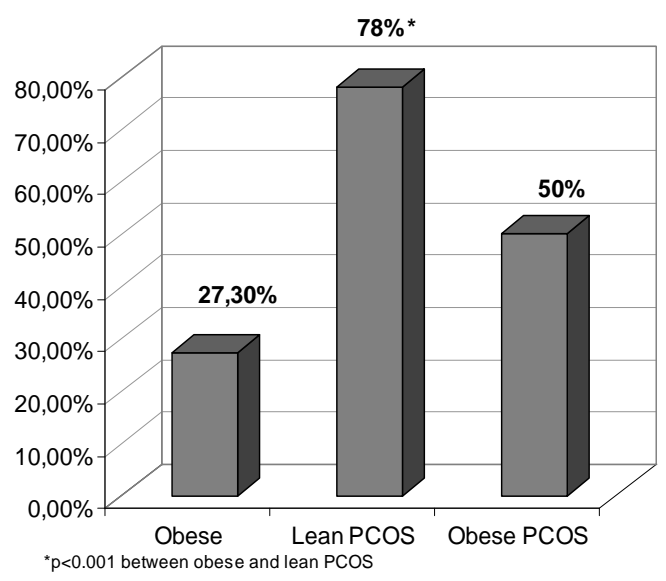

Figure 1. Rate of sexual dysfunction.

Of all androgens FSFI score correlates negatively only with androstednione levels $(\mathrm{r}=-0,356)$ and the correlation is strongest in orgasm domain $(\mathrm{r}=-0,421)$ (Table 3). Women with and without hiperandrogenemia do not show differences in total FSFI score and in desire domain (Figure 2 and Figure 3). It is interesting to note that LH but not FSH shows moderate positive correlation to all domains of FSFI (Table 3).

FSFI scores do not show correlation to the indices of carbohydrate metabolism (blood glucose and IRI during OGTT), lipid profile, arterial pressure and the presence of obstructive sleep apnea and AHI.

After metformin treatment there was an increase in all FSFI domain scores although statistical significance was noted only for the total FSFI score and the domains lubrication and pain (Table 4).

\section{Discussion}

Sexual function is a debated issue in PCOS patients. On the one hand the presence of hirsutism and other signs of hyperandrogenemia and infertility are a potential source of distress and low self esteem but on the other androgens are thought to increase sexual desire. Obesity also has a negative impact on women's sexuality. PCOS women

Table 3. Correlations between total FSFI score and separate domains and androgen levels, LH, FSH and IRI during the OGTT.

\begin{tabular}{|c|c|c|c|c|c|c|c|c|c|c|}
\hline & $\mathrm{LH}$ & FSH & Testo-sterone & Androsten-dione & DHEAS & $\begin{array}{c}17 \mathrm{OH} \\
\text { progesterone }\end{array}$ & IRI $0 \mathrm{~min}$ & IRI $60 \mathrm{~min}$ & IRI $120 \mathrm{~min}$ & HOMA \\
\hline FSFI & $0.353^{*}$ & 0.245 & 0.200 & $-0.356^{*}$ & -0.015 & -0.087 & 0.012 & 0.031 & 0.034 & 0.087 \\
\hline Desire & 0.251 & 0.110 & $0.250 *$ & -0.104 & 0.296 & -0.121 & 0.004 & 0.085 & 0.026 & 0.000 \\
\hline Arousal & $0.304 *$ & 0.242 & 0.167 & $-0.335^{*}$ & -0.032 & -0.095 & 0.061 & 0.066 & 0.005 & 0.090 \\
\hline Lubrication & $0.332 *$ & 0.271 & 0.125 & $-0.339^{*}$ & -0.069 & -0.096 & 0.001 & 0.008 & 0.040 & 0.088 \\
\hline Orgasm & $0.332 *$ & 0.243 & 0.168 & $-0.421^{*}$ & -0.012 & -0.055 & 0.022 & 0.019 & 0.019 & 0.062 \\
\hline Satisfaction & $0.358 *$ & 0.190 & 0.225 & -0.294 & -0.065 & -0.015 & 0.011 & 0.037 & 0.044 & 0.096 \\
\hline Pain & 0.323 & 0.196 & 0.186 & -0.226 & -0.027 & -0.098 & 0.010 & 0.052 & 0.078 & 0.100 \\
\hline
\end{tabular}




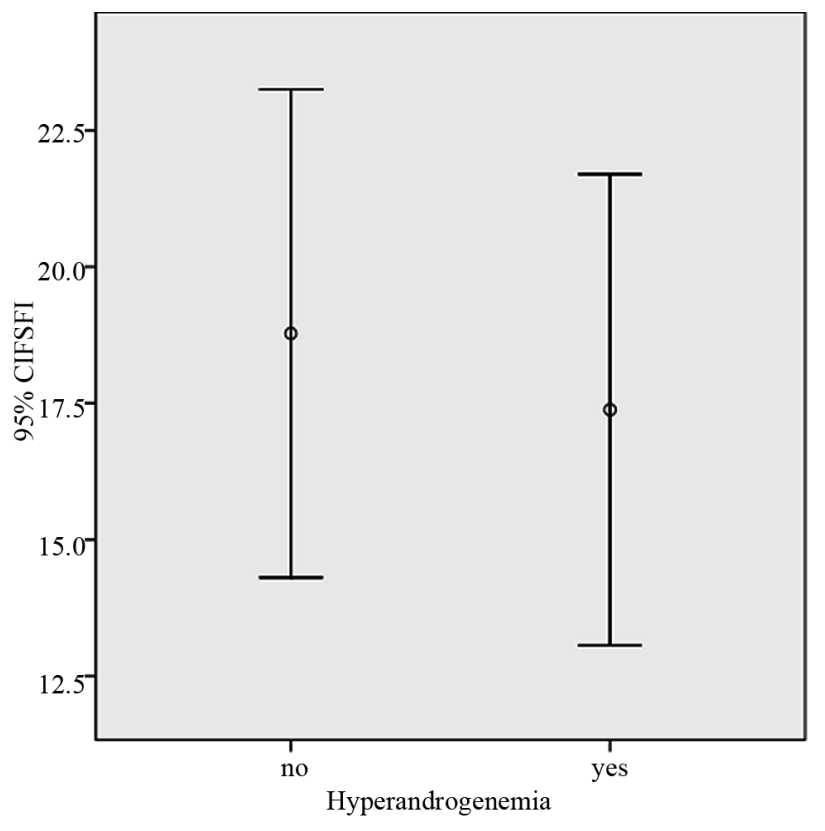

Figure 2. Total FSFI score in patients with and without hyperandrogenemia.

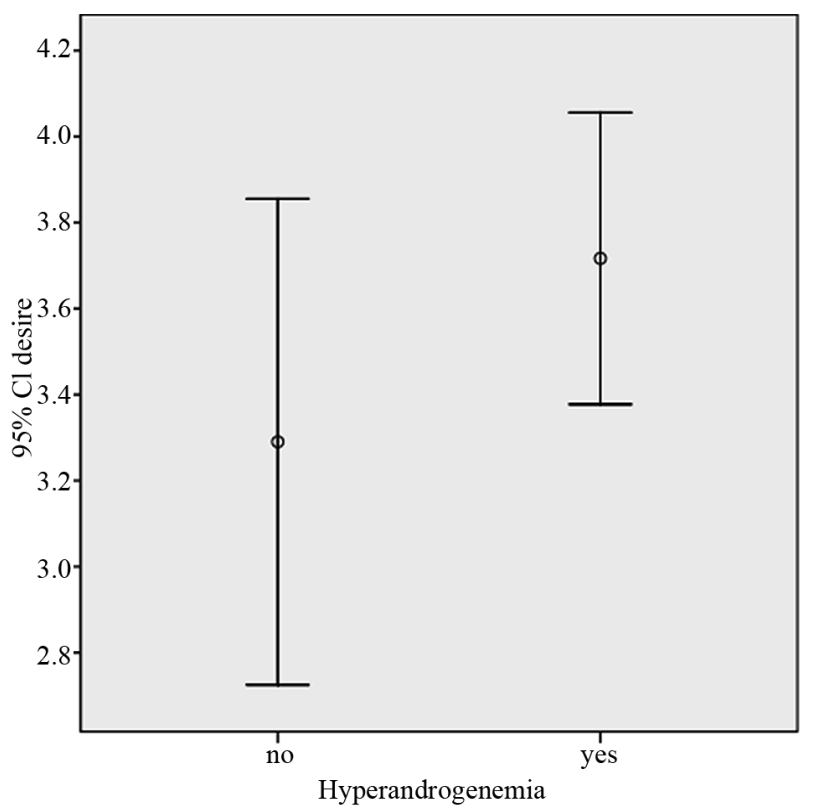

Figure 3. Desire domain score in patients with and without hyperandrogenemia.

seem less satisfied with their sexual life and find themselves less attractive probably due to the high prevalence of overweight and skin manifestations of hypernadrogenism $[10,11]$. In our study however sexual function is not related to androgen levels or to the weight, insulin resistance, dyslipidemia or arterial hypertension. Obese patients without PCOS have much lower rate of sexual dysfunction than PCOS patients with or without obesity. It is possible that the reason for these results is related to
Table 4. Results of FSFI before and after treatment with metformin.

\begin{tabular}{ccc}
\hline & Before treatment & After treatment \\
\hline FSFI & $16.8 \pm 12.6$ & $22.9 \pm 9.8^{*}$ \\
Desire & $3.1 \pm 1.3$ & $3.7 \pm 1.3$ \\
Arousal & $2.7 \pm 2.4$ & $3.7 \pm 2.0$ \\
Lubrication & $2.9 \pm 2.7$ & $4.2 \pm 2.1^{*}$ \\
Orgasm & $2.7 \pm 2.5$ & $3.4 \pm 2.1$ \\
Satisfaction & $2.8 \pm 2.3$ & $3.9 \pm 2.0$ \\
Pain & $2.4 \pm 2.7$ & $4.1 \pm 2.1^{*}$ \\
\hline
\end{tabular}

$* \mathrm{p}<0.05$ difference before and after treatment.

the different mean age of the obese group but we didn't find a significant correlation between the FSFI score and different domains and age. Besides that is has been found that women's sexual function declines with age $[12,13]$. It is a very interesting finding that except desire, the lowest scores on FSFI and the higher rate of sexual dysfunction was observed in lean PCOS subject and not in obese. Desire however is a very important domain of sexual function index and in our study was the lowest in obese PCOS patients probably because of the combination of adverse effect of obesity and clinical signs of PCOS. Androgens are closely related to sexual function in both sexes and have a positive effect on sexual desire (including fantasies) [12]. We however did not find differences in FSFI score or desire domain between women with and without hyperandrogenemia.

Similar to other studies [7], metformin treatment has beneficial effects on sexual function in our study population. The possible reason for this could be related to the effect of metformin on body weight [16], menstrual cycle and androgen levels $[14,15]$. We however did not find a correlation between the change in FSFI score and weight change, change in androgen levels or change in length of menstrual cycle. The results for domains desire, orgasm, arousal and satisfaction however are not statistical signifycant probably because of the small number of patients.

From this study we conclude that:

1) PCOS patients, and in particular the lean ones, have higher rates of sexual dysfunction than obese patients without PCOS.

2) Sexual dysfunction is not related to the age of the patients in the age interval of this study-18 - 45 years.

3) Patients with and without hyperandrogenemia do not have different scores.

4) Metformin treatment has beneficial effect on sexual function in PCOS patients.

\section{REFERENCES}

[1] M. J. Himelein and S. S. Thatcher, "Polycystic Ovary Syndrome and Mental Health: A Reviewm" Obstetrical \& Gynecological Survey, Vol. 61, No. 11, 2006, pp. 723- 


\section{2. doi:10.1097/01.ogx.0000243772.33357.84}

[2] M. E. Trent, M. Rich, S. B. Austin and C. M, "Gordon. Fertility Concerns and Sexual Behavior in Adolescent girls with Polycystic Ovary Syndrome," Journal of Pediatric and Adolescent Gynecology, Vol. 16, No. 1, 2003; pp. 33-37. doi:10.1016/S1083-3188(02)00205-X

[3] J. Bancroft, "Androgens and Sexual Function in Men and Women," In: C. J. Bagatell and W. J. Bremmer, Eds., Androgens in Health and Disease, Humana Press, Totowa, 2003, pp. 258-290. doi:10.1385/1-59259-388-7:259

[4] S. Elsenbruch, S. Hahn, D. Kowalsky, et al., Quality of Life, Psychosocial Well-Being, and Sexual Satisfaction in Women with Polycystic Ovary Syndrome," Journal of Clinical Endocrinology \& Metabolism, Vol. 88, No 12, 2003, pp. 5801-5807. doi:10.1210/jc.2003-030562

[5] C. Battaglia, R. E. Nappi, F. Mancini, et al., "PCOS, Sexuality, and Clitoral Vascularisation: A Pilot Study," The Journal of Sexual Medicine, Vol. 5, No 12, 2008, pp. 2886-2894. doi:10.1111/j.1743-6109.2008.01010.x

[6] H. M. Conaglen and J. V. Conaglen, "Sexual Desire in Women Presenting for Antiandrogen Therapy," Journal of Sex \& Marital Therapy, Vol. 29, No 12, 2003, pp. 255267. doi:10.1080/00926230390195498

[7] S. Hahn, S. Benson, S. Elsenbruch, et al., "Metformin Treatment of Polycystic Ovary Syndrome Improves Health-Related Quality-of-Life, Emotional Distress and Sexuality," Human Reproduction, Vol. 21, No 7, 2006, pp. 1925-1934. doi:10.1093/humrep/del069

[8] M. E. Wierman, R. E. Nappi, N. Avis, S. R. Davis, F. Labrie, W. Rosner and J. L. Shifren, "Endocrine Aspects of Women's Sexual Function," The Journal of Sexual Medicine, Vol. 7, No 1, 2010, pp. 561-585. doi:10.1111/j.1743-6109.2009.01629.x

[9] R. Rosen, C. Brown, J. Heiman, S. Leiblum, C. Meston, R. Shabsign, D. Ferguson and R. D'Agostino, "The Female Sexual Function Index (FSFI): A Multidimens Ional Self-Report Instrument for the Assessment of Female Sexual Function," Journal of Sex \& Marital Therapy, Vol. 26, No. 2, 2000, pp. 191-208. doi:10.1080/009262300278597

[10] O. E. Janssen, S. Hahn, S. Tan, S. Benson and S. Elsenbruch, "Mood and Sexual Function in Polycystic Ovary Syndrome," Seminars in Reproductive Medicine, Vol. 26, No. 1, 2008, pp. 45-52. doi:10.1055/s-2007-992924

[11] G. L. Jones, J. M. Hall, A. H. Balen and W. L. Ledger, "Health-Related Quality of Life Measurement in Women with Polycystic Ovary Syndrome: A Systematic Review," Human Reproduction Update, Vol. 14, No. 1, 2008, pp. 15-25.

[12] S. Siedman, "Hormonal Aspects of Sexual Dysfunction: The Therapeutic Use of Exogenous Androgens in Men and Women," Current Psychiatry Reports, Vol. 2, No. 3, 2000, pp. 215-222. doi:10.1007/s11920-996-0011-5

[13] R. Hayes and L. Dennerstein, "The Impact of Aging on Sexual Function and Sexual Dysfunction in Women: A Review of Population-Based Studies," The Journal of Sexual Medicine, 2005, Vol. 2, No. 3, pp. 317-330. doi:10.1111/j.1743-6109.2005.20356.x

[14] P. Moghetti, R. Castello, C. Negri, F. Tosi, F. Perrone, M. Caputo, E. Zanolin and M. Muggeo, "Metformin Effects on Clinical Features, Endocrine and Metabolic Profiles, and Insulin Sensitivity in Polycystic Ovary Syndrome: A Randomized, Double-Blind, Placebo-Controlled 6-Month Trial, Followed by Open, Long-Term Clinical Evaluation," The Journal of Clinical Endocrinology \& Metabolism, 2000, Vol. 85, No. 1, pp. 139-146. doi: $10.1210 /$ jc. 85.1 .139

[15] K. H. Chou, et al., "Clinical, Metabolic and endocrine Parameters in Response to Metformin in Obese Women with Polycystic Ovary Syndrome: A Randomized, Double-Blind and Placebo-Controlled Trial," Hormonal Aspects of Sexual Dysfunction, Vol. 35, No. 2, 2003, pp. 86-91. doi:10.1055/s-2003-39056

[16] D. A. Haas, B. R. Carr and G. R. Attia, "Effects of Metformin on Body Mass Index, Menstrual Cyclicity, and Ovulation Induction in Women with Polycystic Ovary Syndrome," Fertility and Sterility, Vol. 79, No. 3, 2003, pp. 469-481. doi:10.1016/S0015-0282(02)04800-8 